\title{
A rare clinical case of true hemifacial hyperplasia
}

\author{
Ravi David Austin $^{1}$, MDs, Kumar Chandan Srivastava $^{1}$, MDs, Gopalakrishnan Pranavadhyani ${ }^{2}$, MDS, \\ Deepti Shrivastava ${ }^{3}$, MDS
}

\begin{abstract}
Hemifacial hyperplasia (HFH) is an unusual congenital anomaly of debatable aetiology. It is characterised by facial asymmetry that is caused by a marked unilateral and localised overgrowth of facial soft tissues, bones and teeth. We report a case of true HFH with typical manifestations with a brief review of the clinical features and radiological findings associated with $\mathrm{HFH}$, the differential diagnoses that should be considered and the available treatment options for HFH. It is hoped that this report will supplement the existing literature on this anomaly.
\end{abstract}

Keywords: facial asymmetry, hyperplasia, hypertrophy

\section{INTRODUCTION}

Although the human body is not precisely symmetrical, anomalous variations and asymmetrical growth can occur in the absence of any local cause or general condition. These anomalous changes, which are often not obvious at birth, grow disproportionately and stabilise around the time of puberty or when general skeletal growth ceases. This condition is known as hemihypertrophy, congenital hemihypertrophy, hemimacrosomia, or partial gigantism. It has been reported that hemihypertrophy was first described by Meckel in 1822, and subsequently documented by Wagner in 1839. ${ }^{(1)}$ Hemifacial hyperplasia (HFH), a variant of hemihypertrophy, is described as a rare unilateral congenital anomaly that results in the asymmetric overgrowth of all tissues on the affected side of the face. ${ }^{(2)}$ To the best of our knowledge, only about 50 cases of hemifacial hyperplasia have been reported since it was first described in $1836 .{ }^{(3)}$ The aim of this report is to add to the existing knowledge on true $\mathrm{HFH}$.

\section{CASE REPORT}

A 25-year-old man presented to our institute with complaints of a massive asymptomatic enlargement on the right side of his face since birth. The patient's medical history revealed that the right side of his face had grown slightly faster than the left side throughout childhood and adolescence. The size of the swelling had not increased in the past five years. His prenatal, medical and family histories were noncontributory. Physical examination revealed a healthy, active and mentally alert young man, and a review of his systems was unremarkable.

Oral examination revealed diffuse facial swelling in the right side of his face. The swelling had a smooth surface and extended superiorly to the right lateral aspect of the supraorbital margin (the eyes were spared), inferiorly to the lower border of the mandible, medially to the midline of the face and laterally to the tragus of the ear. The swelling was

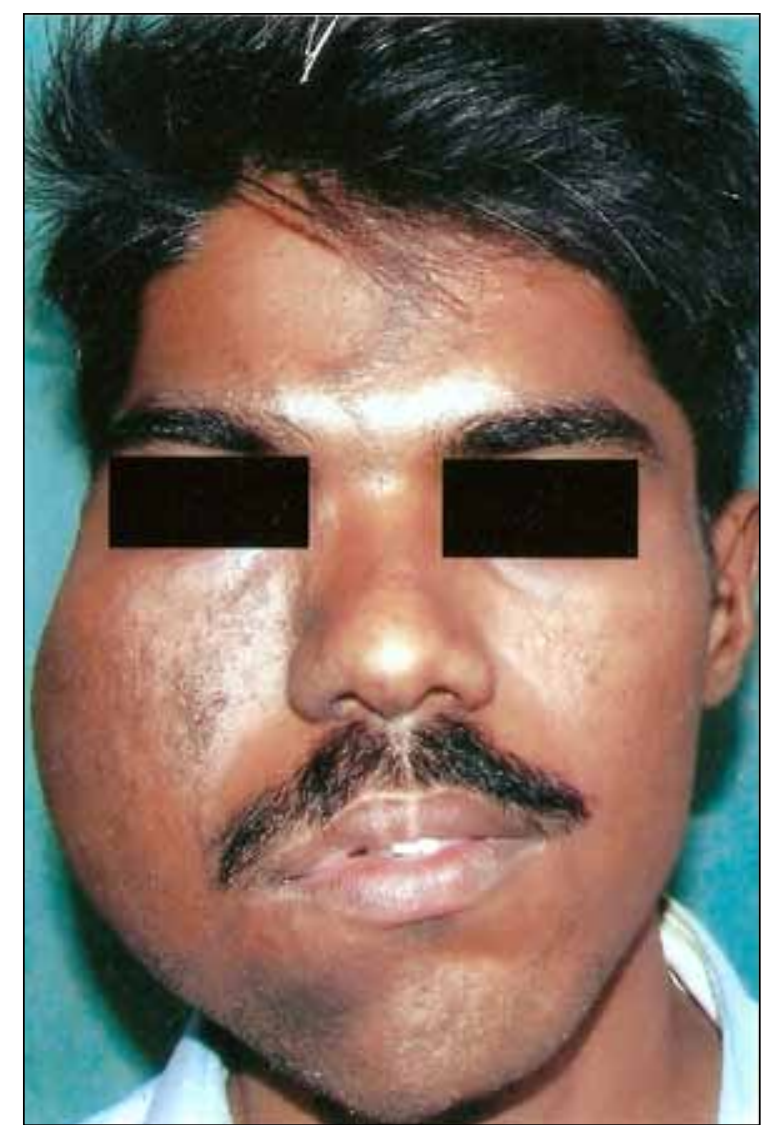

Fig. 1 Photograph shows facial asymmetry due to large and diffuse midfacial swelling. Hyperpigmentation was observed at the middle of the forehead and on the right cheek.

non-tender and hard in consistency. There was no nasal deviation, although his chin had deviated toward the left side of his face. The upper lip on the right side of his face seemed thicker. His skin and hair appeared normal, with the exception of two hyperpigmented, non-hairy patches - one in the middle of the forehead and the other on the cheek (Fig. 1). Examination of the patient's extremities and trunk revealed normal symmetric development.

\footnotetext{
${ }^{1}$ Department of Oral Medicine and Maxillofacial Radiology, Rajah Muthiah Dental College and Hospital, Annamalai University, ${ }^{2}$ Division of Oral Medicine and Radiology, Faculty of Dentistry, J.K.K Natarajah Dental College and Hospital, ${ }^{3}$ Division of Periodontics, Rajah Muthiah Dental College and Hospital, Annamalai University, Tamil Nadu, India

Correspondence: Dr Ravi David Austin, Professor and Head, Department of Oral Medicine and Maxillofacial Radiology, Rajah Muthiah Dental College and Hospital, Annamalai University, Annamalai Nagar, Chidambaram 608001, Tamil Nadu, India. ravidavidaustin@gmail.com
} 


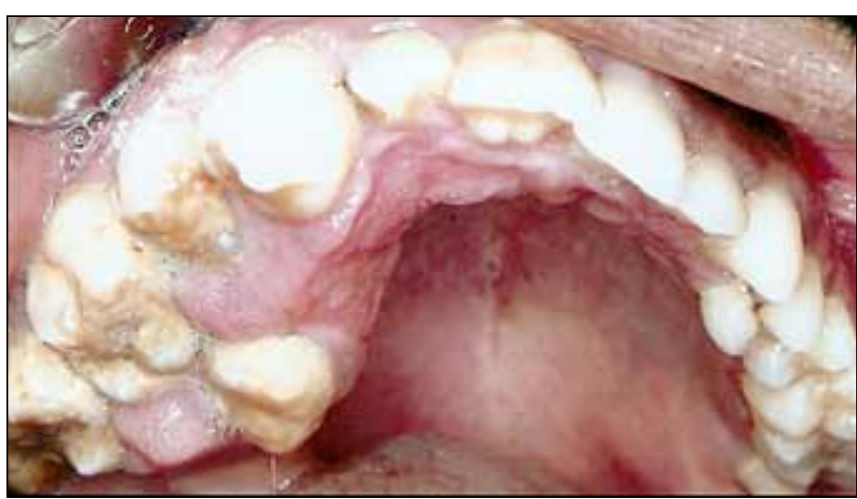

Fig. 2 Intraoral photograph shows an enlarged right maxillary arch.

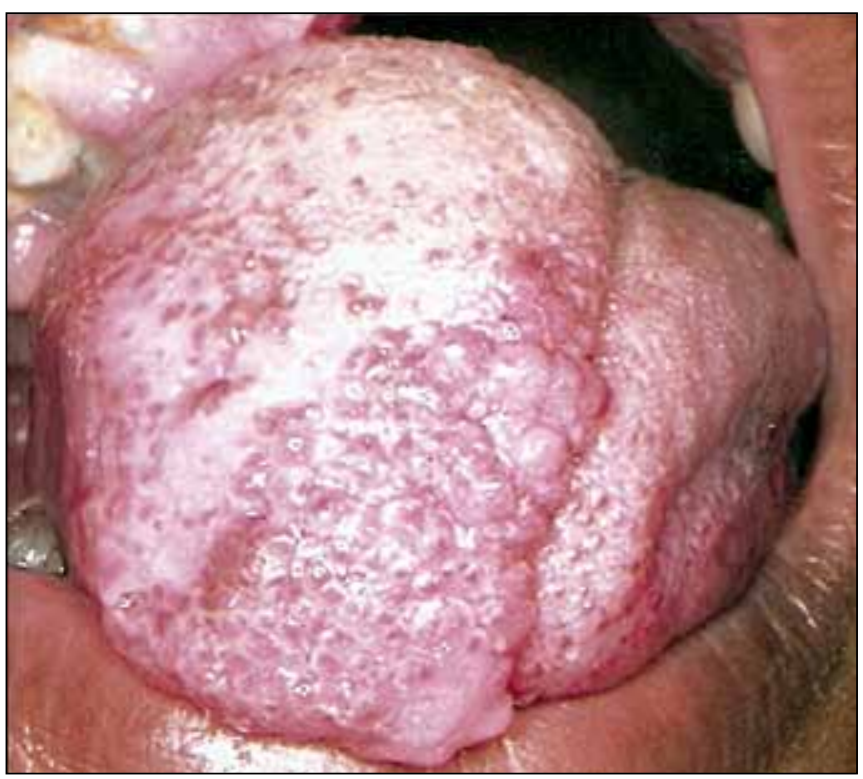

Fig. 3 Photograph shows hyperplasia of the right half of the tongue, with enlargement of the fungiform papillae.

Intraoral examination showed evident asymmetry caused by the enlargement of both the right maxillary and mandibular arches, with no midpalatal deviation (Fig. 2). There was a full complement of upper and lower teeth, except for missing right mandibular first and second molars, which were found to be impacted with incomplete roots on panoramic radiograph. The third molar on the right side was confirmed to be congenitally missing after radiological investigation. The deciduous second molar of the right side was retained. Unilateral macroglossia with typical enlargement of the fungiform papillae was also evident (Fig. 3). Based on the patient's clinical history and features, the condition was provisionally diagnosed as $\mathrm{HFH}$.

Panoramic radiography and axial computed tomography (CT) confirmed the clinical findings. The panoramic radiograph revealed that the right mandibular condyle, coronoid process and mandibular body were enlarged. Significant additional bone growth of the maxillary alveolus, with prominent tuberosity, was also observed (Fig. 4). The observed mandibular deviation toward the left was due to the unilateral enlargement of the body of the right mandible. Increased density was also noticed in the region of the angle of the mandible, symphysis

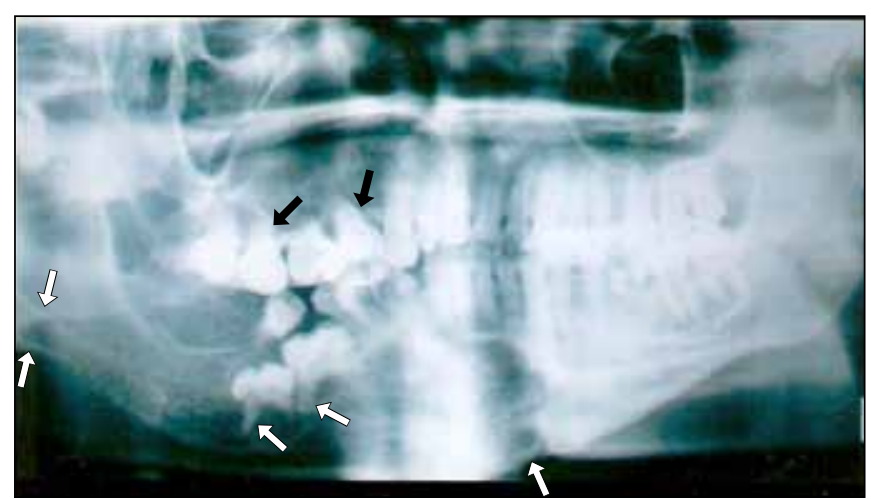

Fig. 4 Panoramic radiograph shows dentition with evidence of rightsided enlarged mandible, macrodontia, widened mandibular canal (white arrows) and blunted roots (black arrows).

menti, as well as the region between the apices of the roots of the right mandibular teeth and the mandibular canal. These features were suggestive of additional bone tissue in the right side of the face. The mandibular canal appeared widened, and alteration in the bony trabeculations was also seen in the right side. Plain CT images revealed that the facial bones, jaw bones, maxillary sinus and associated soft tissues were enlarged (Figs. $5 \& 6$ ). The patient, a teashop owner who was married with apparently normal children, was offered cosmetic surgery, which he declined due to financial constraints.

\section{DISCUSSION}

Primary hemihypertrophy is a rare congenital anomaly characterised by disproportionate growth and gross enlargement of all tissues on one side of the body. ${ }^{(4)}$ Overgrowth of only a portion of an extremity is referred to as segmental hemihypertrophy, while overgrowth of segments on both sides of the body is referred to as crossed hemihypertrophy. ${ }^{(5)}$ In 1962, Rowe classified hemihypertrophy into the following types: (a) simple hemihypertrophy, which involves one or both limbs; (b) complex hemihypertrophy, which involves the entire half of the body; and (c) $\mathrm{HFH}$, which involves the face, head and their associated structures. ${ }^{(6)}$ Rowe further categorised HFH into these two subcategories: (a) true $\mathrm{HFH}$, defined as unilateral enlargement of the viserocranium extending from the frontal bone (excluding the eye) to the lower border of the mandible superoinferiorly and from the midline of the face to the pinna of the ear mediolaterally, with enlargement of all soft tissues, teeth and bone in the area; and (b) partial HFH, in which enlargement is limited to a single structure. Based on Rowe's categorisation, our patient is a case of true $\mathrm{HFH}$.

The literature reports that $\mathrm{HFH}$ was first described by Beck in 1836, ${ }^{(3)}$ and the term 'hemihyperplasia' was suggested to be more appropriate than 'hemihypertrophy' (since the increase in cell number was more significant than the increase in cell volume) by Geiser et al in 1970. (7) Many theories have been formulated in the attempt to suggest a cause for $\mathrm{HFH}$. Some of the causative factors postulated include 


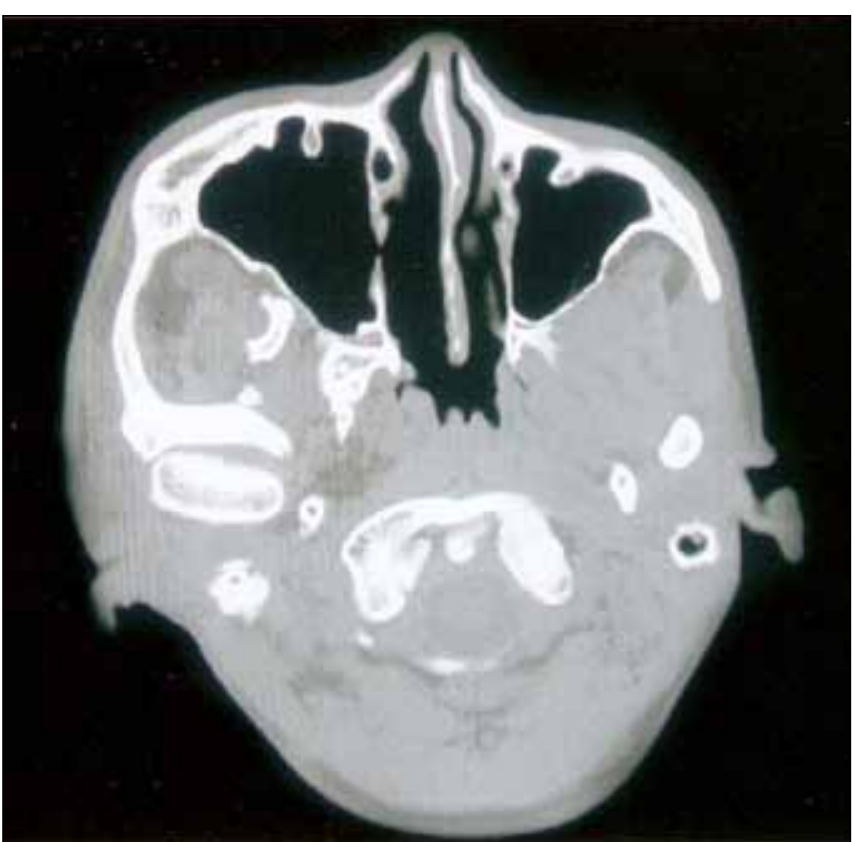

Fig. 5 Plain axial CT image shows enlarged bones, such as the zygomatic bone and condyle, on the right side of the patient's face. Asymmetry in the size of maxillary sinuses was also observed.

biochemical and endocrine dysfunctions, as well as anatomical and functional anomalies of the vascular or lymphatic system (e.g. haemangioma, arteriovenous malformation and lymphangioma). Other proposed theories include disturbances in the development of the branchial $\operatorname{arch}^{(8)}$ and neural tube, ${ }^{(9)}$ and chromosomal abnormalities. ${ }^{(10)}$ In 1944, Rudolph et al reported familial occurrences of $\mathrm{HFH}$ in two cases, and thus postulated heredity as a possible cause. ${ }^{(11)}$

Our patient was male and had right-sided $\mathrm{HFH}$, in agreement with Rowe's literature review, which reported a higher prevalence in males and right-sided facial predominance. ${ }^{(6)}$ In 1965, Ringrose et al reported that asymmetry is usually evident at birth and accentuates with age, especially during puberty.(2) Abnormal enlargement generally ceases at 17 or 18 years of age, which is also around the time of skeletal maturation. ${ }^{(2)}$ A similar chronology of events was reported in our patient. His condition was stable at presentation, and had been stable for five years prior to presentation. This can be correlated to the completion of skeletal maturity.

The extent of asymmetry may vary from an innocuous swelling to a gross distortion. Typically, most patients present with enlargements that are most prominent in the midcheek area and less noticeable in the neck and the region above the level of the zygomatic arch. Most of the clinical findings in our report were typical of the oral and facial characteristics of hemihypertrophy previously reported by various authors, ${ }^{(12,13)}$ particularly with respect to the enlargement of the jaws, teeth, facial soft tissue and tongue on the affected side. Variations in trabecular pattern and changes in the dimensions of the dental arches were also noted in our patient. Furthermore, the patient's palatal vault was strikingly thick

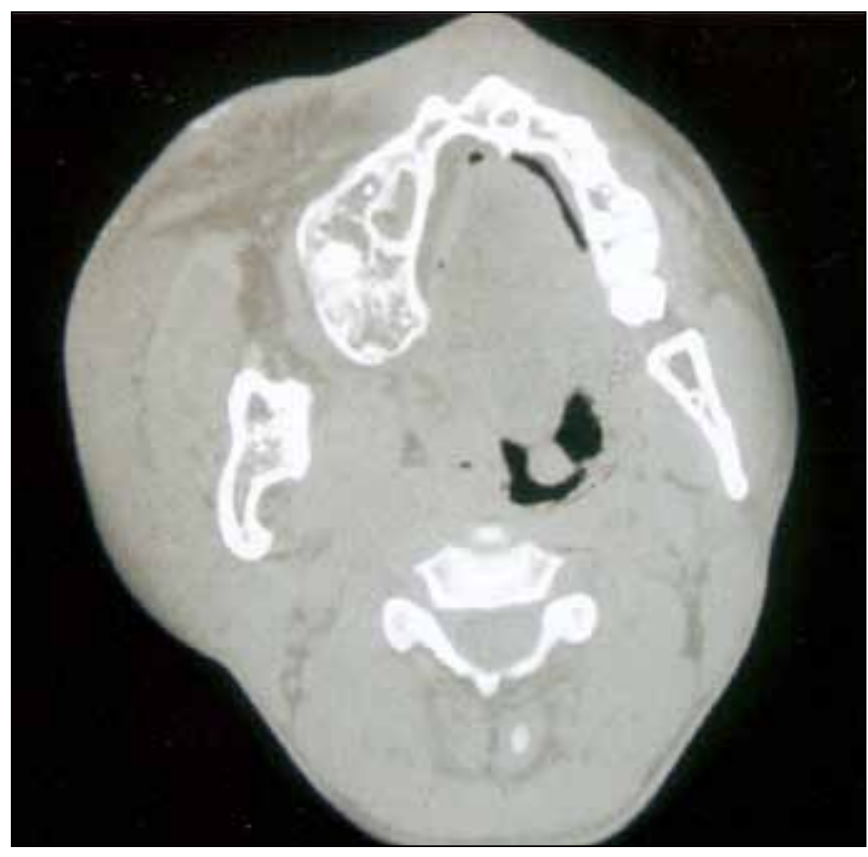

Fig. 6 Plain axial CT image shows increased width of the right maxilla mandible and participation of soft tissues in the facial asymmetry.

on the affected side and well-demarcated at the midline, pushing the midline toward the unaffected left side. The use of the characteristic enlargement of teeth on the affected side as a diagnostic aid in $\mathrm{HFH}$ has been supported by Rushton. ${ }^{(12)}$ It was reported that Rushton analysed 19 patients with hemihypertrophy who had enlarged teeth and found that 15 of them had hemihypertrophy that was limited to the face. ${ }^{(12)}$ Unilateral distribution of dental anomalies (e.g. size and rate of development, crown size and root shape) and concurrent unilateral tongue enlargement with enlarged fungiform papillae are prominent features characteristic of $\mathrm{HFH}$. The identification of most of these pathognomic features in our case led to the diagnosis of $\mathrm{HFH}$.

Precocious eruption and premature development of involved teeth are also well reported by many authors..$^{(1,2,4,12)}$ However, in our case, the right mandibular first and second molars were impacted, with retention of the deciduous second molar on the same side. Malocclusion is a common feature in $\mathrm{HFH}$ due to osseous and dental asymmetries. ${ }^{(13)}$ Asymmetry of the facial bones and associated overlapping soft tissue are some craniofacial findings that also aid in the diagnosis of $\mathrm{HFH} .{ }^{(14)}$ On the affected side, the skin is typically thick and coarse, the hair usually thick and abundant (hypertrichosis), and the sebaceous and sweat gland secretions excessive. Other systems that could be affected by this anomaly include the integumentary, musculoskeletal, cardiovascular, neurological, genitourinary, endocrine and respiratory systems. ${ }^{(1,4,5)}$

When evaluating a patient suspected to have $\mathrm{HFH}$, numerous entities have to be considered in the differential diagnosis. Congenital causes of cranial asymmetry include a variety of syndromes such as Beckwith-Weidemann, Proteus, McCune-Albright, epidermal naevus, Klippel-Trénaunay and 
Maffucci syndromes. However, all of these aforementioned syndromes have distinguishing features other than body asymmetry. Other conditions that manifest at an early age such as neurofibromatosis, hyperpituitarism and infiltrating lipomatosis are largely bilateral and widespread. ${ }^{(13)}$ Acquired causes of cranial asymmetry include Romberg syndrome, vascular anomalies and fibro-osseous lesions. Romberg syndrome is characterised by an acquired unilateral underdevelopment, with muscle weakness and neurological deficit. ${ }^{(15)}$ Vascular anomalies (e.g. haemangioma) and multiple arteriovenous aneurysms of the bone are distinguished by their characteristic clinical and investigatory (e.g. Doppler ultrasonography, magnetic resonance imaging and angiography) findings. ${ }^{(13)}$ Fibro-osseous lesions (e.g. fibrous dysplasia, Paget's disease and ossifying fibroma) and other bony tumours usually have no soft tissue component and may have serum chemistry changes. ${ }^{(14)}$

Generally, treatment of HFH is for cosmetic purposes. Emotional support and counselling during the developing years is important in order to help the individual cope, as reconstructive procedures such as soft tissue debulking, face lifts, osteotomies and/or orthognathic surgery can only be planned and performed when growth ceases. ${ }^{(4)}$

In conclusion, congenital hemihypertrophy is a rare disorder with varied manifestations. Its presentation may range from the enlargement of a single digit to that of the entire half of the body. In this report, we presented a clinical case of HFH that had most of the pathognomic features used to diagnose $\mathrm{HFH}$ - unilateral enlargement of the viserocranium and enlargement of all tissues (teeth, bone and soft tissue) on the affected side.

\section{REFERENCES}

1. Islam MN, Bhattacharyya I, Ojha J, et al. Comparison between true and partial hemifacial hypertrophy. Oral Surg Oral Med Oral Pathol Oral Radiol Endod 2007; 104:501-9. Epub 2007 Apr 20.

2. Antoniades K, Letsis I, Karakasis D. Congenital hemifacial hyperplasia. Br J Oral Maxillofac Surg 1988; 26:344-8.

3. Khanna JN, Andrade NN. Hemifacial hypertrophy. Report of two cases. Int J Oral Maxillofac Surg 1989; 18:294-7.

4. Horswell BB, Holmes AD, Barnett JS, Hookey SR. Primary hemihypertrophy of the face: review and report of two cases. J Oral Maxillofac Surg 1987; 45:217-22.

5. Wilson FC Jr, Orlin H. Crossed congenital hemihypertrophy associated with a Wilm's tumor; report of a case. J Bone Joint Surg Am 1965; 47:1609-14.

6. Rowe NH. Hemifacial hypertrophy. Review of literature and addition of four cases. Oral Surg Oral Med Oral Pathol 1962; 15:572-87.

7. da Silveria EJ, Godoy GP, Lins RD, de Medeiros AM, Queiroz LM. Partial facial hemihyperplasia with 9 years of evolution: case report and review of literature. Oral Surg Oral Med Oral Pathol Oral Radiol Endod 2006; 102:501-5

8. Lee S, Sze R, Murakami C, Gruss J, Cunningham M. Hemifacial myohyperplasia: description of a new syndrome. Am J Med Genet 2001; 103:326-33.

9. Pollock RA, Newman MH, Burdi AR, Condit DP. Congenital hemifacial hyperplasia: an embryologic hypothesis and case report. Cleft Palate J 1985; 22:173-84.

10. Cohen MM Jr. Perspectives on craniofacial asymmetry. IV. Hemiasymmetries. Int J Oral Maxillofac Surg 1995; 24:134-41.

11. Rudolph CE, Norvold RW. Congenital partial hemihypertrophy involving marked malocclusion. J Dent Res 1944; 23:133-9.

12. Lawoyin JO, Daramola JO, Lawoyin DO. Congenital hemifacial hypertrophy. Report of two cases. Oral Surg Oral Med Oral Pathol 1989; 68:27-30.

13. Sapp JP, Eversole LR, Wysocki GP. Contemporary Oral and Maxillofacial Pathology. St Louis: Mosby, 2004.

14. Regezi JA, Sciubba JJ. Oral Pathology: Clinical Pathologic Correlations. 4th ed. Philadelphia: Saunders, 2003.

15. Mazzeo N, Fisher JG, Mayer MH, Mathieu GP. Progressive hemifacial atrophy (Parry-Romberg syndrome). Case report. Oral Surg Oral Med Oral Pathol Oral Radiol Endod 1995; 79:30-5. 